\title{
Effective state of polarization of a photon-beam
}

\author{
Łukasz Michalik, Andrzej W. Domański* \\ Faculty of Physics, Warsaw University of Technology, Koszykowa 75, 00-662 Warszawa
}

Received February 28, 2011; accepted March 11, 2011; published March 31, 2011

\begin{abstract}
In the paper a quantum description of an effective state of polarization for an electromagnetic field containing variously polarized photons is presented. The discussion of the problem includes the quantum character of a single photon, comparison with a classical field, matrix description of photon polarization and physical interpretation of the Pauli representation of a density operator that represents photons in a statistical ensemble.
\end{abstract}

In quantum optics, the description of an electromagnetic field is based on quantum oscillators and each of them is connected with a different direction of light propagation, different polarization of light $(\mu=1,2)$ and different energy of transition in an atom $(\Delta E=\hbar \omega)$. Each act of emission/absorption in an atom is related to changing the excitation level in an oscillator because it physically means that one photon appears/disappears. The state of an oscillator excited $n$ times is created from the vacuum state |0) [1]:

$$
|n\rangle=\frac{\left(\hat{a}^{+}\right)^{n}}{\sqrt{n !}}|0\rangle,
$$

where $|n\rangle$ is the eigenstate of an energy operator, which can be expressed by annihilation $(\hat{a})$ and creation $\left(\hat{a}^{+}\right)$ operators or by photon-number operator $(\hat{N})[1]$ :

$$
\begin{aligned}
& \hat{H}=\frac{\hat{p}^{2}}{2 m}+\frac{1}{2} m \omega^{2} \hat{x}^{2}= \\
& =\left(\hat{a}^{+} \hat{a}+\frac{1}{2}\right) \hbar \omega=\left(\hat{N}+\frac{1}{2}\right) \hbar \omega .
\end{aligned}
$$

Although the photon is usually depicted as a wavepacket or some kind of "elementary piece" of an electromagnetic field, it is not trivial to make a connection between state vector $|n\rangle$ (which is Schrödinger's equation solution) and time-space distribution $\mathbf{E}(\mathbf{r}, t)$ of the field.

It can be proved that the phase of $\mathbf{E}(\mathbf{r}, t)$ and the number of photons satisfy the uncertainty principle [2]:

\footnotetext{
*E-mail: domanski@if.pw.edu.pl
}

$$
\Delta n \cdot \Delta \varphi \geq \frac{1}{2}
$$

In particular, it means that $\mathbf{E}(\mathbf{r}, t)$ cannot be written for single photons. Each statistical realization of a field connected with photon emission is completely unpredictable. It automatically means that the photon does not have any determined spectrum.

The plane wave is related to a so-called coherent state, which is the superposition of Hamiltonian eigenstates [2]:

$$
|\alpha\rangle=\exp \left(-\frac{|\alpha|^{2}}{2}\right) \cdot \sum_{n=0}^{\infty} \frac{\alpha^{n}}{\sqrt{n !}}|n\rangle,
$$

where $\alpha$ is a complex amplitude of a wave. Quantity $\omega$ takes on a meaning of frequency in the case of coherent states. The number of photons is a random variable described by the Poisson probability distribution:

$$
P_{n}=|\langle n \mid \alpha\rangle|^{2}=\exp \left(-|\alpha|^{2}\right) \cdot \frac{\alpha^{2 n}}{n !}
$$

The electromagnetic field in a coherent state has similar properties to classical waves. For example, if one considers the behaviour of that field on a beam-splitter there is no quantum entanglement at the output, in contrast to $|n\rangle$ states [2].

Further concern of various effects is simple, because plane waves with different frequencies can be added classically. Thus one can see complementarity to the well known theory of temporal partial coherence of light, in particular - the value of a degree of polarization (DOP) less than 1, which can be understood as a consequence of mismatching states of polarization for different wavelengths.

Let us return to Hamiltonian eigenstates of a quantum oscillator. When we want to define the state of polarization for a single photon, we consider the state vector [3]: 


$$
|\psi\rangle=c_{1}|\mathrm{~h}\rangle+c_{2}|\mathrm{v}\rangle
$$

where $|\mathrm{h}\rangle=\hat{a}_{\mu=1}^{+}(\mathbf{k})|0\rangle$ and $|\mathrm{v}\rangle=\hat{a}_{\mu=2}^{+}(\mathbf{k})|0\rangle$ are horizontal and vertical polarizations of a photon. Thus $|\psi\rangle$ is a superposition of these two types of oscillator's excitation. Coefficients $c_{i}$ may be complex values, in general. They satisfy the normalization condition. Because of temporal evolution of Hamiltonian eigenstates [4]:

$$
|\psi(t)\rangle=e^{-3 i \omega t / 2}\left(c_{1}|\mathrm{~h}(0)\rangle+c_{2}|\mathrm{v}(0)\rangle\right),
$$

it is reasonable to write the phase factor $e^{i \varphi_{r}}$ only by one of the coefficients - then it also has the meaning of a (constant) phase difference between $c_{1}$ and $c_{2}$. Using the Dirac (vector) notation, one can write:

$$
|\psi\rangle=\left|\theta, \varphi_{r}\right\rangle=\left[\begin{array}{c}
\cos \left(\frac{\theta}{2}\right) \\
\sin \left(\frac{\theta}{2}\right) e^{i \varphi_{r}}
\end{array}\right] .
$$

Form (8) is consistent with the general rule that density operator $\hat{\rho}=|\psi\rangle\langle\psi|$ is a Hermitian matrix. It can be further represented as a sum of identity matrix $\hat{\sigma}_{0}$ and Pauli matrices $\hat{\sigma}_{i}$ with coefficients $s_{i}(i=1,2,3)$ [2]:

$$
\hat{\rho}=\frac{1}{2}\left(\hat{\sigma}_{0}+\sum_{i=1}^{3} s_{i} \hat{\sigma}_{i}\right)
$$

The coefficients from the above representation form a socalled Bloch vector:

$$
[\mathbf{s}]=\left[\begin{array}{c}
s_{0} \\
s_{1} \\
s_{2} \\
s_{3}
\end{array}\right]=\left[\begin{array}{c}
1 \\
\sin \theta \cdot \cos \varphi_{r} \\
\sin \theta \cdot \sin \varphi_{r} \\
\cos \theta
\end{array}\right],
$$

which is depictured on the Bloch sphere. It is similar to the Stokes vector and Poincare sphere but the main difference is that linearly polarized states (which are basis states) are at the sphere poles. Because of some aspects in describing quantum filters [3], the angle ranges should be chosen as: $\theta \in\langle 0 ; 2 \pi)$ and $\varphi_{r} \in\langle 0 ; \pi)$. A few examples of polarizations are:
- $\left|+45^{0}\right\rangle=\left|\frac{\pi}{2}, 0\right\rangle=\frac{1}{\sqrt{2}}(|\mathrm{~h}\rangle+|\mathrm{v}\rangle)$ is $+45^{0}$ linear polarization

- $\left|-45^{0}\right\rangle=\left|\frac{3 \pi}{2}, 0\right\rangle=\frac{1}{\sqrt{2}}(|\mathrm{~h}\rangle-|\mathrm{v}\rangle)$ is $-45^{0}$ linear polarization

- $|+\rangle=\left|\frac{\pi}{2}, \frac{\pi}{2}\right\rangle=\frac{1}{\sqrt{2}}(|\mathrm{~h}\rangle+i|\mathrm{v}\rangle)$ is left handed circular polarization

- $|-\rangle=\left|\frac{3 \pi}{2}, \frac{\pi}{2}\right\rangle=\frac{1}{\sqrt{2}}(|\mathrm{~h}\rangle-i|\mathrm{v}\rangle)$ is right handed circular polarization

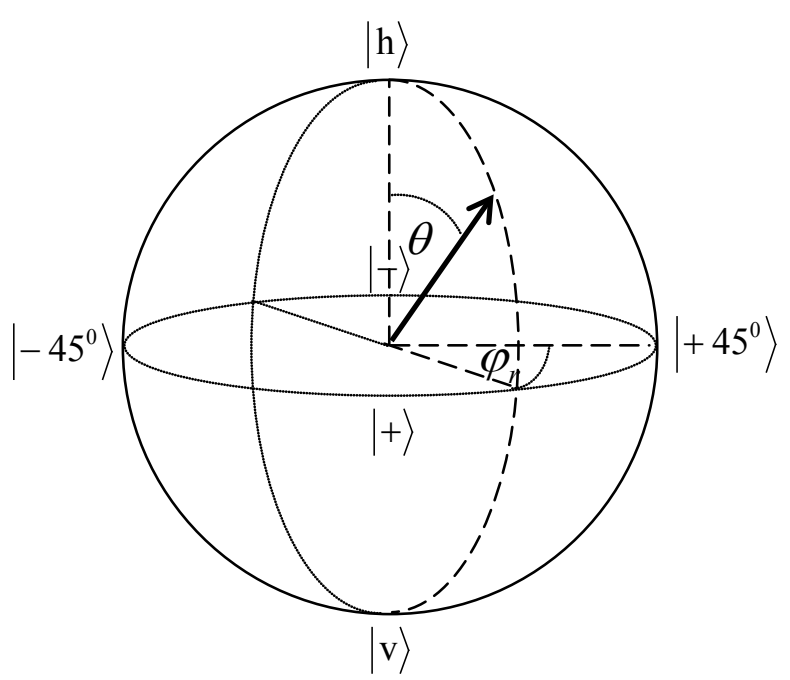

Fig. 1. Bloch sphere with examples of photon polarizations.

The quantum field containing a determined number of photons which are variously polarized and propagate in the same direction in free space (let us call it a photonbeam) need to be described by using a mixed quantum state:

$$
\hat{\rho}_{m i x}=\int_{0}^{2 \pi \pi} \int_{0} P\left(\theta, \varphi_{r}\right)\left|\theta, \varphi_{r}\right\rangle\left\langle\theta, \varphi_{r}\right| \sin \theta d \theta d \varphi_{r}
$$

where $P\left(\theta, \varphi_{r}\right)$ is the probability density distribution that the state of polarization of a random photon picked from a statistical ensemble is $\left|\theta, \varphi_{r}\right\rangle$. The double integral is a sum over all angles' values and $\sin \theta$ appears as a result of coordinate system transformation. 
The density operator for a mixed state can be also represented as a sum of the identity matrix and Pauli matrices with proper coefficients, but these coefficients do not form a normalized vector as in the case of Eq. (10). Thus it may be written as [2]:

$$
\hat{\rho}_{m i x}=\frac{1}{2}\left(\hat{\sigma}_{0}+p \cdot \sum_{i=1}^{3} s_{i}^{e f} \hat{\sigma}_{i}\right)
$$

where $\quad|p|<1$ and $\sum_{i=1}^{3}\left(s_{i}^{e f}\right)^{2}=1$. After elementary transformations (11) takes the form [5]:

$$
\hat{\rho}_{\text {mix }}=\frac{P_{1}}{2}\left(\hat{\sigma}_{0}+\sum_{i=1}^{3} s_{i}^{e f} \hat{\sigma}_{i}\right)+\frac{P_{2}}{2}\left(\hat{\sigma}_{0}-\sum_{i=1}^{3} s_{i}^{e f} \hat{\sigma}_{i}\right)
$$

where $p=\frac{P_{1}-P_{2}}{P_{1}+P_{2}}, \quad P_{1}+P_{2}=1$. Both sums in Eq. (13) represents states vectors on the Bloch sphere, so the density operator can be rewritten as:

$$
\begin{aligned}
& \hat{\rho}_{m i x}=P_{1} \cdot\left|\theta_{e f}, \varphi_{e f}\right\rangle\left\langle\theta_{e f}, \varphi_{e f}\right|+ \\
& +\left(1-P_{1}\right) \cdot\left|\pi+\theta_{e f}, \varphi_{e f}\right\rangle\left\langle\pi+\theta_{e f}, \varphi_{e f}\right|
\end{aligned}
$$

It is the key conclusion - both of the representations (11) and (14) are equivalent. It means that the sum over all possible states boils down to the sum of only two states which have opposite senses on the Bloch sphere. Representing one of the states as $\left|\theta_{e f}, \varphi_{e f}\right\rangle$ makes it a socalled effective state of polarization. Angle parameters that describe it can be calculated when we rewrite Eq. (11) and (14) in matrix forms and compare :

$$
\hat{\rho}_{\operatorname{mix}}=\int_{0}^{2 \pi \pi} \int_{0} P\left(\theta, \varphi_{r}\right)\left[\begin{array}{cc}
\cos ^{2}\left(\frac{\theta}{2}\right) & \frac{1}{2} \sin \theta \cdot e^{-i \varphi_{r}} \\
\frac{1}{2} \sin \theta \cdot e^{i \varphi_{r}} & \sin ^{2}\left(\frac{\theta}{2}\right)
\end{array}\right] \sin \theta d \theta d \varphi_{r}
$$

and

$$
\hat{\rho}_{m i x}=\left[\begin{array}{cc}
\frac{1}{2}(p+1) \cos \theta_{e f}+\sin ^{2}\left(\frac{\theta_{e f}}{2}\right) & \frac{1}{2} p \sin \theta_{e f} \cdot e^{-i \varphi_{e f}} \\
\frac{1}{2} p \sin \theta_{e f} \cdot e^{i \varphi_{e f}} & -\frac{1}{2}(p+1) \cos \theta_{e f}+\cos ^{2}\left(\frac{\theta_{e f}}{2}\right)
\end{array}\right] .
$$

This comparison leads us to four simultaneous equations and $\theta_{e f}, \varphi_{e f},|p|$ are the solution:

$$
\left\{\begin{array}{l}
\operatorname{tg} \varphi_{e f}=\frac{B}{A} \\
\operatorname{tg} \theta_{e f}=\frac{\sqrt{A^{2}+B^{2}}}{C} \\
|p|=\sqrt{A^{2}+B^{2}+C^{2}}
\end{array}\right.
$$

where:

$$
\begin{aligned}
& \int_{0}^{2 \pi} \int_{0} P\left(\theta, \varphi_{r}\right) \cdot \sin ^{2} \theta \cdot \cos \varphi_{r} d \theta d \varphi_{r}=A, \\
& \int_{0}^{2 \pi \pi} \int_{0} P\left(\theta, \varphi_{r}\right) \cdot \sin ^{2} \theta \cdot \sin \varphi_{r} d \theta d \varphi_{r}=B, \\
& \frac{1}{2} \int_{0}^{2 \pi \pi} \int_{0}^{2 \pi} P\left(\theta, \varphi_{r}\right) \cdot \sin (2 \theta) d \theta d \varphi_{r}=C .
\end{aligned}
$$

In conclusion, we presented a new way to describe the photon state in a statistical ensemble. The equations above show the relation between an effective state of polarization and probability density distribution $P\left(\theta, \varphi_{r}\right)$ - this function characterizes the photon source that emits one photon by another, forming a photon-beam.

\section{References}

[1] R.J. Glauber, Quantum Theory of Optical Coherence (Wiley-VCH 2007).

[2] C.C. Gerry, P.L. Knight, Introductory Quantum Optics (Cambrige Univeristy Press 2005)

[3] R.J. Glauber, New Techn. Ideas Quant. Measur. Theory 480, 336 (1986).

[4] L. Michalik, J. Jasinski, P.L. Makowski, A.W. Domanski, Proc. SPIE 7745, 7745OK (2010).

[5] T. Tudor, A. Gheondea, Jour. Opt. Soc. Am. A 24, 204 (2007). 